\title{
The Hippocampus Maps Concept Space, Not Feature Space
}

\author{
${ }^{-1}$ Stephanie Theves, ${ }^{1,2}$ Guillén Fernández, ${ }^{2}$ and Christian F. Doeller ${ }^{1,3}$ \\ ${ }^{1}$ Max-Planck-Institute for Human Cognitive and Brain Sciences, 04103 Leipzig, Germany, ${ }^{2}$ Donders Institute for Brain, Cognition, and Behaviour, \\ Radboud University and Radboud University Medical Center, 6525 EN Nijmegen, The Netherlands, and ${ }^{3}$ Kavli Institute for Systems Neuroscience, \\ Centre for Neural Computation, The Egil and Pauline Braathen and Fred Kavli Centre for Cortical Microcircuits, NTNU, Norwegian University of \\ Science and Technology, NO-7491 Trondheim, Norway
}

The hippocampal formation encodes maps of space and a key question in neuroscience is whether its spatial coding principles also provide a universal metric for the organization of nonspatial, conceptual information. Previous work demonstrated directional coding during navigation through a continuous stimulus feature space as well as mapping of distances in a feature space that was relevant for concept learning. Here we provide the first unambiguous evidence for a hippocampal representation of the actual concept space, by showing that the hippocampal distance signal selectively reflects the mapping of specifically conceptually relevant rather than of all feature dimensions. During fMRI scanning of 32 human participants (21 females), we presented everyday objects, which had beforehand been associated with specific values on three continuous feature dimensions. Crucially, only two dimensions were relevant to prior concept learning. We find that hippocampal responses to the objects reflect their relative distances in a space defined along conceptually relevant dimensions compared with distances in a space defined along all feature dimensions. These findings suggest that the hippocampus supports knowledge acquisition by dynamically encoding information in a space spanned along dimensions that are relevant in relation to define concepts.

Key words: conceptual knowledge; fMRI; hippocampus; learning; spatial coding

Significance Statement

How are neural representations of conceptual knowledge organized, such that humans are able to infer never experienced relations or categorize new exemplars? Map-like representations as supported by the hippocampal formation to encode physical space during navigation have been suggested as a suitable format. Here we provide the first evidence for a hippocampal representation of a conceptual space compared with a general feature-based space.

\section{Introduction}

The role of the hippocampus in concept learning is subject to debate (Knowlton and Squire, 1993; Zaki, 2004; Kumaran, 2012). Concepts are organizing structures that define how contents are related to each other and can be used to transfer meaning to novel input (Smith and Medin, 1981; Kemp, 2012). Their formation thus inherently depends on generalization over, and

\footnotetext{
Received Mar. 1, 2020; revised July 14, 2020; accepted July 15, 2020.

Author contributions: S.T. designed research; S.T. performed research; S.T. analyzed data; S.T., G.F., and C.F.D. wrote the paper.

This work was supported by the Netherlands Organization for Scientific Research (Grant NWO-Gravitation 024-001-006). C.F.D. is supported by the Max Planck Society; the Kavli Foundation; the European Research Council (Grant ERC-COG GEOCOG 724836); the Center of Excellence scheme of the Research Council of Norway - Center for Neural Computation (Grant 223262/F50); The Egil and Pauline Braathen and Fred Kavli Center for Cortical Microcircuits; the National Infrastructure scheme of the Research Council of NorwayNORBRAIN (Grant 197467/F50); and the Netherlands Organization for Scientific Research (Grants NW0-Vidi 452-12-009; NWO-MaGW 406-14-114; and NWO-MaGW 406-15-291).

The authors declare no competing interests.

Correspondence should be addressed to Stephanie Theves at theves@cbs.mpg.de or Christian F. Doeller at doeller@cbs.mpg.de.

https://doi.org/10.1523/JNEUROSCI.0494-20.2020

Copyright $(2020$ the authors
}

integration of experiences. Thus, a role of the hippocampus in generalization seemed considerable because of its roles in binding elements into spatial and episodic context (Davachi et al., 2003; Davachi, 2006; Ranganath, 2010; Komorowski et al., 2013) as well as integration of information over episodes (Davis et al., 2012; Collin et al., 2015; Milivojevic and Doeller, 2013; Milivojevic et al., 2015; Schlichting et al., 2015; beyond the episodic and spatial domain: Mack et al., 2016; Theves et al., 2019). Specifically, previous studies reported an involvement of the hippocampus in categorization (Nomura et al., 2007; Zeithamova et al., 2008; Davis et al., 2012; Mack et al., 2013; Seger et al., 2015; Kim et al., 2018). How specific this involvement is with regard to the conceptual aspect of the task and how the hippocampus, as opposed to other brain regions, supports the acquisition of conceptual knowledge remained unclear. A recent proposal is that map-like organization of new information by the hippocampalentorhinal system similar to mental representations of space (O'Keefe and Dostrovsky, 1971; Hafting et al., 2005; Morgan et al., 2011; Howard et al., 2014; Horner et al., 2016), might be specifically suited to explain inference of not directly experienced relations (c.f. inferring shortcuts during navigation) (Behrens et al., 2018), as well 
as the transfer of meaning to novel information via localization of new input in the conceptual map. However, spatial coding principles have yet never been unambiguously linked to concept learning. So far, evidence for spatial coding in nonspatial domains has been limited to nonspatial feature dimensions without a direct link to conceptual relevance: Electrophysiological recordings in rodents demonstrated the involvement of place and grid cells in coding sound frequency during an auditory discrimination task (Aronov et al., 2017), and human fMRI studies showed both a directional, grid cell-like signal in entorhinal cortex during active navigation through a stimulus feature space (Constantinescu et al., 2016), as well as a hippocampal representation of distances in multidimensional feature space that was relevant to concept learning (Theves et al., 2019). Specifically, in the latter study, participants acquired a concept of two stimulus categories, which was defined in two-dimensional space along the feature dimensions of the stimuli, via a categorization task. Hippocampal representations of distances in concept space were measured via responses to passively viewed objects that had before been associated with specific stimuli (i.e., positions in concept space). As the associated stimuli only included features that defined the space of the concept, the important question remained whether the hippocampus maps the objects according to all feature dimensions of their associated stimuli (feature space mapping) or specifically for the purpose of concept learning (concept space mapping). Here we aim to distinguish between these two accounts by orthogonally manipulating conceptual and featurebased relationships between objects during learning. In sum, we show that the hippocampal responses to objects reflect the twodimensional distances between objects that emerge from their positions in concept space, compared with distances that emerge in a space including an integration of the conceptually irrelevant feature dimension.

\section{Materials and Methods}

\section{Experimental design and subject details}

Thirty-two healthy students (mean age: $23 \pm 3$ years; 21 females) from the Radboud University campus participated in this study. All participants were right handed and had normal or corrected-to-normal vision. All participants gave written informed consent and were financially compensated for participation. The study was approved by the local ethics committee (CMO Arnhem-Nijmegen, The Netherlands).

\section{Design}

In a learning phase, participants acquired a novel concept of two abstract stimulus categories which was defined within a two-dimensional space along two of three stimulus feature dimensions with the diagonal through the two-dimensional space serving as category boundary (Fig. 1). The third stimulus feature was irrelevant to categorization and did thus not contribute to the concept space. Participants further learned to associate six everyday objects with six specific abstract stimuli. Here, associated abstract stimuli had to be memorized precisely in all three feature dimensions. Now critically, before and immediately after the learning phase, the everyday objects were presented in the MRI scanner to test whether hippocampal responses to the objects correspond specifically to their two-dimensional conceptual distances rather than to their three-dimensional distances emerging in the full feature space. To distinguish between concept- and feature-based mapping, objects were positioned such that their two- and three-dimensional distance relations were uncorrelated (Pearson's $r=0.1$ ).

\section{Stimuli}

The experiment involved the following two sets of stimuli: everyday objects (generated with the video game Sims; www.thesims3.com) and abstract stimuli (generated via MATLAB 2014a; Fig. 1B, associations). The abstract stimuli varied along the following three stimulus feature dimensions: opacity, frequency of dots, and frequency of stripes. On each dimension, abstract stimuli could vary along 10 steps, resulting in a total stimulus space of 1000 feature combinations. Step sizes on each of the three feature dimensions were evaluated psychophysically before the experiment to assure comparable discriminability of all three dimensions.

\section{Procedures}

The study took place on $1 \mathrm{~d}$. A learning phase in which the conceptual context of six objects was acquired over the course of four tasks (details below), was preceded and followed by object-viewing blocks (OVB) in the scanner (Fig. 1). During the OVB, participants performed a targetobject detection task (orthogonal to any conceptual content) to assure attention to the stimuli.

\section{Object viewing block}

Images of seven objects, of which six where used in the learning phase and one served as a catch-object, were presented in a pseudorandomized sequence with a stimulus duration of $1 \mathrm{~s}$ and interstimulus intervals of 3.5, 5, and $6.5 \mathrm{~s}$ (33.3\% each). Participants were instructed to indicate for each object whether it is a trampoline (i.e., a catch object) or not, using a button box (buttons counterbalanced across participants). The task included 246 trials (236 for participant 10) with a catch trial rate of $12 \%$. Each object was presented equally often.

The learning phase comprised four tasks in the following order: associative learning, 3D reconstruction, categorization, and navigating concept space, all before the post-learning OVB. The post-learning OVB was followed by a final 3D recall test (Fig. 1).

\section{Associative learning}

Associations between the six objects presented in the OVB and specific abstract stimuli had to be learned in alternating encoding and test blocks. The assignment of objects to abstract stimuli was randomized across participants, such that measuring hippocampal responses to the objects during scanning enabled us to read out their conceptual distances rather than visual or semantic similarities. In the encoding blocks, objects were presented next to their corresponding abstract stimulus and participants were instructed to memorize the presented pairs. Participants were told that they will need to memorize the associations in all their features throughout the entire experiment. The presentation order of the six pairs was pseudorandomized with each object/stimulus being equally often presented on the left/right position of the screen. Pairs were presented for $2 \mathrm{~s}$ on the screen and each pair was shown three times per encoding block. Each encoding block was followed by a test block in which the object is presented in the center of the screen along with the six abstract stimuli displayed (in a randomized order) below the object. Every association was tested once in blocks 1-6 and twice from block 7 onward in a randomized order. Participants selected the abstract stimulus associated with the presented object via key press (1-6) and received feedback $(500 \mathrm{~ms})$ on whether the choice was correct. Participants underwent at least eight encoding and test blocks (i.e., 60 test trials), and beyond that were trained until exceeding $90 \%$ accuracy over all previous test trials. An upper limit of 168 test trials was set because of the limited time between the prescheduled fMRI sessions.

\section{$3 D$ reconstruction}

Encoding and recognition of associations was followed by a free recall. Participants were instructed to precisely recall the abstract stimulus associated with a presented object, and subsequently adjust a start stimulus in all three feature dimensions until it matches the associated stimulus. A trial could only be completed by adjusting all three dimensions correctly. Each of the six abstract stimuli had to be reconstructed once. Dimensions were upregulated and downregulated using six adjacent keys. 
A

$\begin{gathered}\text { Object viewing } \\ \text { block (fMRI) }\end{gathered}$
$\longrightarrow$

B

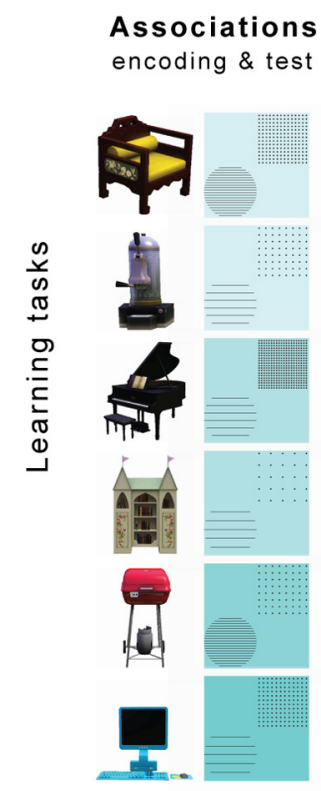

3D reconstruction

recall associations

"Reconstruct the symbol associated with..until correct."

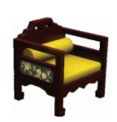

Categorization
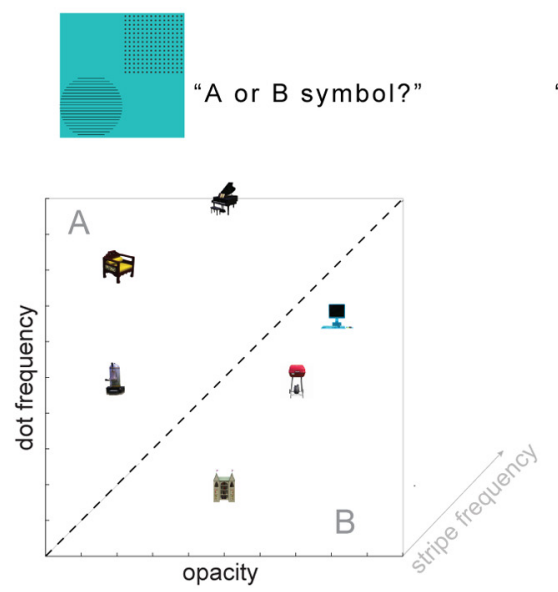

Navigating concept space

"Collect category A object"

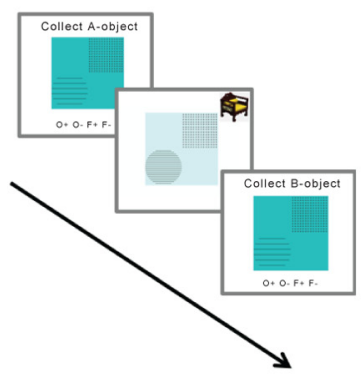

D

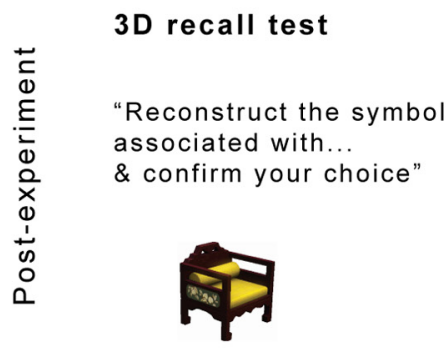

Figure 1. Experimental design. $\boldsymbol{A}$, Learning (for details, see $\boldsymbol{B}$ ) was framed by OVBs inside the scanner to measure emerging neural representations (for details, see $\boldsymbol{C}$ ). A final $3 \mathrm{D}$ recall test probed memory across feature dimensions (for details, see $\boldsymbol{D}$ ). $\boldsymbol{B}$, Between OVBs, the following four learning tasks were completed (order: left to right): (1) participants learned six associations between objects and three-dimensional stimuli via encoding and test blocks, and (2) freely recalled them by reconstructing the 3D stimulus associated with a given object until correct; (3) subsequently, they acquired the concept of two stimulus categories via feedback-based categorization along the diagonal in two-dimensional concept space; and (4) navigating concept space required the $2 \mathrm{D}$ reconstruction (third dimension randomized) of an object-associated stimulus of a certain category. $C$, In the OVBs, the six objects plus an additional catch object were presented pseudorandomly with a stimulus duration of $1 \mathrm{~s}$ and interstimulus intervals of $3.5,5$, and $6.5 \mathrm{~s}$ while participants performed a catch object detection task. $\boldsymbol{D}$, During postexperimental assessment of memory across feature dimensions, participants had to reconstruct the 3D stimulus associated with a given object and confirm their choice.

\section{Categorization}

Participants were instructed to categorize abstract stimuli (see Stimuli) into two categories (A and B symbols), based on the relation of the opacity and dot frequency of a stimulus, whereas stripe frequency was unpredictive of category membership. Categories were, unbeknown to the participant, delineated via the diagonal (Fig. 1, dashed line) through a two-dimensional space spanned by the two relevant feature dimensions (2D concept space). As training stimuli, we selected for each participant a subset of 720 stimuli from the total stimulus space (1000 possible 3D feature combinations), including all possible off-diagonal combinations of opacity and dot frequency with randomly selected stripe frequency values. Stimuli were presented in a randomized sequence. In each trial, one abstract stimulus was presented in the center of the screen, and its category had to be selected via key press. Participants were given a maximum of $6 \mathrm{~s}$ to respond, and each response was followed by feedback $(500 \mathrm{~ms})$. Categorization training included at least 300 trials and afterward stopped when accuracy exceeded $85 \%$ across all previous trials or the maximal number of 720 trials (set because of time constraints between fMRI sessions) was reached. Instructions did not include any indications of a spatial rule.

We did not define an absolute performance criterion for "associative learning" and "categorization" since we expected high across-subject variance in both tasks (based on previous and pilot work), and time was constrained given the learning phase taking place in between two prescheduled fMRI sessions. We also did not opt for a fixed number of trials to avoid unnecessary training in one task when a participant would have needed more trials to achieve high performance in the other task. Instead, with the present combination of trial limit and performance criterion, we intended to optimize the division of training time between these two tasks on an individual-subject level. Importantly, the term "criterion" does not refer to the exclusion criterion (this instead was based on the actual chance level (16.6\% in associative learning, $50 \%$ in categorization). 
Navigating $2 D$ concept space

In each trial, an abstract stimulus (selected from the total pool of 1000 possible 3D combinations) was presented and participants were instructed to "collect" an object of a certain category (i.e., "Collect an Aobject") by editing the two feature dimensions that were relevant for category membership (using four adjacent keys). Thus, they had to combine their knowledge of the specific object-stimulus pairs with categorical rule knowledge. A trial was completed when they navigated to one of the object "locations" that met the category-specific ratio of opacity and dot frequency and the collected object appeared together with its associated 3D stimulus on the screen. The third, conceptually irrelevant dimension had not to be adjusted by the participants. Instead, it was randomized across trials and remained constant within a trial. Once a participant correctly adjusted opacity and dot frequency to one of the required object locations, also the third feature switched to the respective value, serving as a reminder of the $3 \mathrm{D}$ associations relevant in subsequent tasks. There were 30 different start positions: 15 in category A, 15 in category B. Targets (Collect A-object or Collect B-object) were assigned pseudorandomly to the start positions, such that in half of the trials participants had to change the category field, while in the other half they did not. The task comprised 60 trials. Participants were instructed to collect each of the six objects at least seven times. The rationale behind the task was to familiarize participants with the conceptual context of the objects. Importantly, no distance relationships between the objects were introduced through this process, because participants did not navigate between the locations of the objects but started from random positions in the feature space.

\section{$3 D$ recall test (subsequent to postlearning OVB)}

We wanted to assure, that adjusting only the conceptually relevant dimensions during navigation did not result in a better memory of these two over the third feature of the six object-associated stimuli. Thus, on being cued by an object, participants had to adjust the three features of a start stimulus to match the stimulus associated with the object and eventually confirm their choice to enter the next trial. Each abstract stimulus had to be constructed four times. This allowed us to compare error rates in recall accuracy of the three feature dimensions. One participant did not conduct this task.

All tasks were conducted using Presentation 16.4 (NBS), except the $3 \mathrm{D}$ reconstruction, Navigation, and 3D recall tasks, which were programmed using Anaconda 2.7 (Python).

\section{MRI methods}

All images were acquired using a 3T PrismaFit MR scanner equipped with a 32-channel head coil (Siemens). A 4D multiband sequence (84 slices; multislice mode; interleaved; voxel size, $2 \mathrm{~mm}$ isotropic; $\mathrm{TR}=1500 \mathrm{~ms} ; \mathrm{TE}=28 \mathrm{~ms}$; flip angle $=65^{\circ}$, acceleration factor $\mathrm{PE}=2$; $F O V=210 \mathrm{~mm}$ ) was used for functional image acquisition. In addition, a structural T1 sequence (MPRAGE, $1 \mathrm{~mm}$ isotropic; TE $=3.03 \mathrm{~ms}$; $\mathrm{TR}=2300 \mathrm{~ms}$; flip angle $=8^{\circ} ; \mathrm{FOV}=256 \times 256 \times 192 \mathrm{~mm}$ ) was acquired. Separate magnitude and phase images were acquired to create a gradient field map (multiband sequence with voxel size of $3.5 \times 3.5 \times 2.0 \mathrm{~mm}$; $\mathrm{TR}=1020 \mathrm{~ms} ; \mathrm{TE}=10 \mathrm{~ms}$; flip angle $=45^{\circ}$ ).

Preprocessing of functional images was performed with FSL 5.0.9. Motion correction and high-pass filtering at $100 \mathrm{~s}$ was applied to the functional datasets. The following exclusion criteria for excessive motion were applied: mean absolute displacement $>2 \mathrm{~mm}$; or peak in absolute displacement $>4 \mathrm{~mm}$; mean \pm SD of absolute displacement of the analyzed sample: $0.427 \pm 0.205 \mathrm{~mm}$ (before) and $0.438 \pm 0.199 \mathrm{~mm}$ (after). The FSL brain extraction toolbox was used to create a skull-stripped structural image. The structural scans were downsampled to $2 \mathrm{~mm}$ (matching the functional image resolution) and segmented into gray matter, white matter (WM), and CSF. Spatial smoothing (Gaussian) was performed at $3 \mathrm{~mm}$. Mean intensity values at each time point were extracted for WM and used as nuisance regressors in the general linear model (GLM) analyses (see below). Structural images were registered to the MNI template. For each functional dataset (pre-learning, postlearning), the preprocessed mean image was registered to the individual structural scan and the MNI template. The coregistration parameters of the mean functional image were applied to all functional volumes.

\section{Statistical analyses}

fMRI data analysis: first level GLMs

All GLMs (GLM 1-2) included regressors accounting for catch trials and button presses as well as six motion parameters as covariates.

$2 D$ versus $3 D$ : Distances between objects in the two-dimensional concept and three-dimensional feature space were modeled in the same GLM (GLM 1), using a stimulus onset regressor indicating the onset and duration of an object on the screen and two regressors being parametrically weighted by the two-dimensional and three-dimensional distances between an object to the preceding object, respectively. Distances between objects in either space were calculated given the feature-based coordinates of the associated stimuli on the respectively relevant dimensions. Smaller distances were expected to result in lower signals, reflecting fMRI adaptation. We calculated the contrast between the two- and three-dimensional distance regressors ( $2 \mathrm{D}$ vs $3 \mathrm{D}$ contrast).

$2 D$ versus $2 D$ (irrelevant) dimensionality control: If a potential difference in the $2 \mathrm{D}$ versus $3 \mathrm{D}$ contrast (GLM 1) would be merely because of a difference in dimensionality (i.e., a coding preference of the hippocampus for two dimensions) rather than because of a difference in conceptual relevance, two-dimensional distances in concept space (2D $x y$ ) should not explain the hippocampal signal better than two-dimensional distances derived from a combination with the conceptually irrelevant $z$ axis (2D $x z, 2 \mathrm{D} y z)$. Thus, we ran a GLM (GLM 2) with all three twodimensional distance predictions as regressors $(2 \mathrm{D} x y, 2 \mathrm{D} x z, 2 \mathrm{D} y z)$ and contrasted the $2 \mathrm{D} x y$ regressor against both alternative $2 \mathrm{D}$ regressors (2D $x z, 2 \mathrm{D} y z)$. Resulting $\beta$-maps were transformed to MNI space to extract the average $\beta$ value of each ROI for subsequent analysis.

\section{fMRI data analysis: group-level analyses}

First-level contrasts of the $\beta$ estimates of the distance regressors were each averaged across all voxels within an ROI for each participant, and the distribution of these values was tested for significance (at $\alpha=5 \%$ ) using one-sample permutation $t$ tests (Groppe, 2010) in which 1000 random permutations were computed to estimate the distribution of the null hypothesis. Correction for multiple comparisons for the number of spatial models tested [main analysis (GLM1): models 2D, 3D; post hoc dimensionality control analysis (GLM 2): models 2D $x y, 2 \mathrm{D} x z, 2 \mathrm{D} y z]$ were performed using the t-max method (Blair and Karniski, 1993). Because of clear directed predictions on the relations between fMRI adaptation and distance (e.g., decreasing distance was supposed to be reflected in a higher fMRI adaptation, following the study by Theves et al., 2019), one-sided tests were applied. To test for effects on the wholebrain level, individual contrasts of the $2 \mathrm{D}$ versus $3 \mathrm{D}$ comparison were subjected to the second-level analysis. Cluster extend-based thresholding $(z=3.1, p=0.05)$ was performed to correct for multiple comparisons.

\section{ROI definition}

For the hippocampal ROI mask, we thresholded probability maps from the Harvard-Oxford structural cortical atlas of the hippocampus at $50 \%$ probability.

\section{Results}

\section{Behavior}

\section{Object detection task (fMRI session)}

The six objects that were associated with abstract stimuli during learning plus an additional catch object were presented multiple times in a randomized sequence that was identical between the prelearning block and the postlearning block. Participants indicated via button press whether or not a presented object was the catch object. The task was performed with high accuracy (percentage of correct responses: pre-learning (mean \pm SD): $98.28 \pm 3016 \%$; post-learning: $98,018 \pm 1991 \%$ ), indicating that participants paid attention to the objects. 


\section{Learning tasks}

Associative learning. Associations between objects and abstract stimuli were studied in alternating encoding and test blocks. Participants performed between 60 and, maximally, 168 test trials (mean \pm SD: $137.313 \pm 40.689$ trials), and within that range training was terminated on reaching accurate performance in $90 \%$ of all previous trials (see rationale for criterion in Procedures). The average final accuracy level was $87.315 \pm$ 7.993\% across all participants. Fifteen participants who did not fully reach the criterion within the trial limit were just short of $90 \%$ accuracy in the final trial $(82.537 \pm 9.710 \%)$. Thus, all participants exceeded chance level (i.e., 16.6\%) by far.

$3 D$ reconstruction. The six object-to-abstract stimulus associations were each recalled once in a $3 \mathrm{D}$ reconstruction task, in which on an object cue, the associated stimulus had to be reconstructed by adjusting all three feature dimensions to the correct value. Each trial ended on correct completion. An ANOVA comparing deviation of "different coordinates visited" from "required steps" across dimensions $(F=11.8, p<0.0001$; post hoc paired $t$ tests: $x$ vs $y: p=0.013, t_{(31)}=2.739 ; x$ vs $z: p=0.001, t_{(31)}=4.391$; $y$ vs $\left.z: p=0.115, t_{(31)}=1.608\right)$ shows that editing dimension $z$ was accomplished with fewer unnecessary edits compared with dimension $x$ and equally well relative to dimension $y$, indicating that the later conceptually irrelevant dimension $z$ was initially encoded. The ultimate knowledge of all three dimensions at the end of learning and critical time of scanning is, however, appropriately captured by the final $3 \mathrm{D}$ recall test.

Categorization. Participants learned to categorize abstract stimuli within at least 300 , but maximally 720 feedback-based trials $(542.906 \pm 205.428$ trials). Within this range, training stopped when $85 \%$ of all previous trials had been classified correctly. Across all participants, the average accuracy was $82.892 \pm 4.639$. Sixteen participants narrowly missed $85 \%$ accuracy in their final trial $(79.158 \pm 3.488 \%)$. Thus, all participants performed considerably above chance-level (i.e., 50\%).

Navigating $2 \mathrm{D}$ concept space. Categorical knowledge as well as knowledge about the six object associations had to be combined in a subsequent "navigation in concept space." Here each trial required collecting an object of a certain category by adjusting the two conceptually relevant feature dimensions until a category-specific object location was reached. All objects were on average collected at least seven times.

$3 D$ recall test (postscanning). Subsequent to the final scanning session, recall accuracy of all three dimensions of the abstract stimuli was tested in a $3 \mathrm{D}$ reconstruction task that required participants to confirm their adjustments as soon as they considered them correct. Recall errors [deviation of reconstructed value from actual coordinate; opacity (10): $0.440 \pm 0.472$; frequency dots $(y)$ : $0.427 \pm 0.474$; frequency stripes $(z)$ : $0.701 \pm 0.725$; $n=31]$ did not differ across dimensions $\left(F_{(2,92)}=2.44\right.$, $p=0.0932 ; n=31$; post hoc pairwise tests between relevant and irrelevant dimensions: $x$ vs $z: t_{(30)}=-1.710, p=0.109 ; y$ vs $\left.z: t_{(30)}=-1.792, p=0.091\right)$.

\section{fMRI}

Hippocampal signal reflects distances in a 2D concept space, not in a $3 D$ feature space

We hypothesized that the hippocampus supports the formation of conceptual knowledge by organizing novel information in a space defined along conceptually relevant dimensions. Following the study by Theves et al. (2019), we expected distances between objects in an abstract space defined along stimulus feature dimensions to be reflected in fMRI adaptation in which the distance to the preceding object would scale with the strength of the hippocampal response (smaller distances relate to higher similarity of the neural response pattern and thus in higher adaptation). The representation of feature-based distances reported in the study by Theves et al. (2019) was shown to be specific to the hippocampus (no effects in whole brain or in ROI analyses on control regions: lateral occipital cortex, postcentral gyrus, entorhinal cortex). Here, we aim to further examine this hippocampal distance effect by probing whether hippocampal responses to the objects are explained specifically by distances between objects in a concept space (defined only along the two conceptually relevant stimulus feature dimensions) or by distance predictions derived from a space defined along all three stimulus dimensions in feature space. We found that hippocampal adaptation significantly scaled with distances between objects in the two-dimensional concept space, but not with distances derived from the full three-dimensional feature space (2D: $t_{(31)}=3.090, p=0.003$; $3 \mathrm{D}$ : $t_{(31)}=-1.434, p=0.916$; corrected for multiple comparisons; see Materials and Methods). The two-dimensional conceptual distances also explain the hippocampal response significantly better than the three-dimensional feature-based distances (contrast 2D vs $3 \mathrm{D}$ : $t_{(31)}=3.163, p=0.001$; Fig. $2 B$ ). We did not observe significant $2 \mathrm{D}$ versus $3 \mathrm{D}$ effects in other brain regions (whole-brain cluster-extend-based thresholding, $z=3.1, p=0.05$ ).

\section{The $2 \mathrm{D}$ concept versus $3 \mathrm{D}$ feature space contrast does not reflect} differences in dimensionality

If the better fit of the hippocampal response by the two-dimensional (vs the three-dimensional) distances would merely reflect a difference in dimensionality (i.e., a coding preference of the hippocampus for two dimensions) rather than a difference in conceptual relevance between both spaces, two-dimensional distances in concept space $(x y)$ should not explain the hippocampal signal better than two-dimensional distance predictions derived from a combination with the conceptually irrelevant feature dimension [i.e., the opacity-dot frequency $(x z)$ plane or stripe frequency-dot frequency $(y z)$ plane]. Thus, we constructed a GLM with 2D $x y$ (concept space), 2D $x z$, and 2D $y z$ as regressors. Only the 2D distance in concept space, but none of the alternative $2 \mathrm{D}$ models integrating $z$, predicts the hippocampal signal $\left[2 \mathrm{D}(x y): t_{(31)}=2.678, p=0.010 ; 2 \mathrm{D}(x z): t_{(31)}=0.268, p=0.497\right.$; $2 \mathrm{D}(y z): t_{(31)}=1.569, p=0.847$; corrected for multiple comparisons]. The 2Dxy (concept space) also reveals a significantly stronger adaptation than the $2 \mathrm{D}$ controls $\left(2 \mathrm{D} x y\right.$ vs $2 \mathrm{D} x z: t_{(31)}=$ $2.439 p=0.015 ; 2 \mathrm{D} x y$ vs $2 \mathrm{D} y z: t_{(31)}=2.786, p=0.004$; corrected for multiple comparisons; Fig. 2C).

\section{Discussion}

For the first time, we demonstrate a direct link between concept learning and hippocampal representations of abstract spaces defined by nonspatial dimensions. While the hippocampus had before been shown to encode distances in a multidimensional feature space as a result of concept learning (Theves et al., 2019), we here intended to discriminate whether this representation reflects the complete feature space or specifically the space embedding the concept. In our design, object relationships could be described in a two-dimensional concept space defined along only conceptually relevant stimulus dimensions and in a threedimensional feature space defined along all stimulus dimensions. We found that hippocampal representations of objects encountered during prior concept learning reflected their concept- and not their feature-based distances. This effect could not be attributed to the difference in dimensionality between concept and 

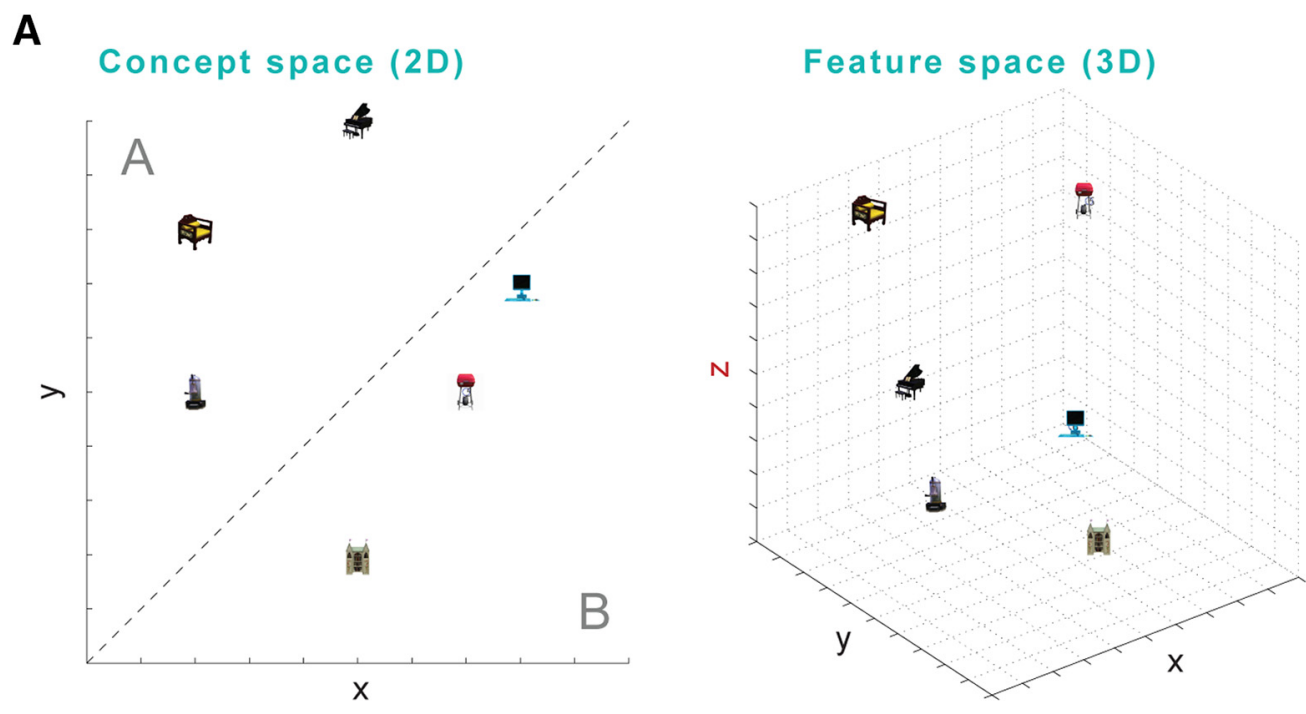

B

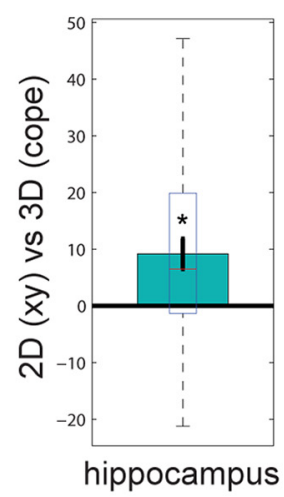

C

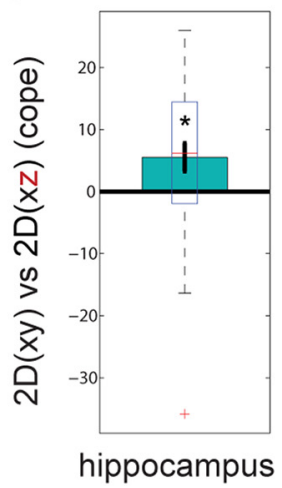

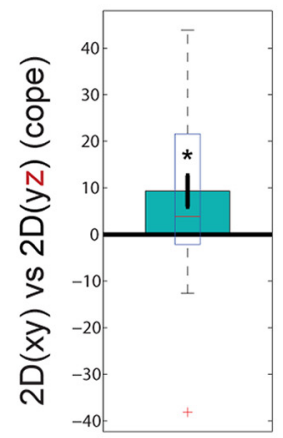

hippocampus

Figure 2. Hippocampal distance code for concept space revealed by fMRI adaptation. $\boldsymbol{A}$, Schematic of two-dimensional object positions and distances between objects in concept space (left) and three-dimensional object positions and distances between objects in feature space (right). B, Average of contrast of parameter estimates (cope) of the 2D versus 3D distance adaptation regressors in all hippocampal voxels. Hippocampal adaptation decreases with increasing two-dimensional conceptual distance between successively presented objects significantly more than with three-dimensional feature-based distance between objects. C, Control for complexity difference between two- and three-dimensional representation: two-dimensional distances from conceptual space $(x y)$ were compared with two-dimensional distances derived from a combination of the conceptually relevant $x$-axis (left) and $y$-axis (right) with the irrelevant $z$-axis. If the better fit of $2 \mathrm{D}(x y)$ distances versus $3 \mathrm{D}$ distances $(\boldsymbol{B})$ reflects a preference of the hippocampus for $2 \mathrm{D}$ codes, $2 \mathrm{D}(x y)$ should not fit better than $2 \mathrm{D}(x z)$ or $2 \mathrm{D}(y z)$. Bars reflect the mean. Central marks of the boxes indicate the median, the bottom and top edges of the boxes indicate the 25th and 75th percentiles, whiskers extend to extreme data points not considered outliers; outliers are plotted as red crosses. Asterisk (*) indicates significance at $p<0.05$.

feature space predictions and a potential coding preference of the hippocampus for two dimensions, as the two-dimensional distances in concept space also explained the hippocampal signal significantly better than alternative two-dimensional distances derived from combinations with the conceptually irrelevant feature dimension. Thus, the hippocampal signal reflects only a representation of distances in a space spanned by the dimensions that were relevant in relation to each other to define the concept, while the mnemonically, but not conceptually, relevant third dimension was not integrated in a multidimensional representation. In sum, we show that the hippocampus organizes new information in a map-like representation in support of concept learning.

First, this suggests that during concept learning, the hippocampus actively organizes new information in a multidimensional space according to conceptually relevant dimensions, and not according to any perceptually present information. The notion that hippocampal spatial codes might not involve incidental sensory information is also in accordance with recent investigations in rodents (Aronov et al., 2017). In the context of the current study, it should further be noted that the better fit of concept- over feature-based distances to the hippocampal signal also speaks against the distance representation being a secondary effect, reflecting the similarity of the sensory input to the hippocampus during potential pattern completion to the associated three-dimensional stimuli.

The present effect further distinguishes between conceptual and general task relevance: all three feature dimensions are task relevant with respect to the mnemonic component of the learning phase. Specifically, concept learning (i.e., categorization) requires setting two feature dimensions in relation to each other, making a map-like representation that integrates both dimensions advantageous. Thus, regarding the question of whether spatial codes in the hippocampus are domain general, it is conceivable that the hippocampus organizes information along arbitrary dimensions (spatial or abstract) into map-like representations as long as the dimensions are relevant in relation to each other (Eichenbaum, 2004; navigation in or representation of 2D spaces; Constantinescu et al., 2016; Bao et al., 2019; Theves et al., 2019). Regarding the role of the hippocampus in concept 
learning in particular, hippocampal spatial codes can be considered a candidate mechanism that is specifically suited to address the typically relational nature of conceptual knowledge (i.e., knowing which features and relations between them distinguish different categories).

Despite evidence for three-dimensional spatial coding in the hippocampus (Yartsev and Ulanovsky, 2013; Kim et al., 2017; Porter et al., 2018; Wohlgemuth et al., 2018), it is currently unknown whether the hippocampus would map a 3D concept space if three dimensions were conceptually relevant. Accordingly, one might speculate on whether a potential hippocampal preference for two dimensions favors the $2 \mathrm{D}$ over the $3 \mathrm{D}$ model. Critically, if this would be the only reason for the better fit of the two-dimensional distances to the hippocampal signal, while there are no differences regarding the integration of feature dimensions in a combined representation, we would expect the two-dimensional distances derived from combinations with the conceptually irrelevant dimension to be encoded in the same way as the twodimensional conceptual distances and thus to likewise fit the hippocampal response. We ruled out this alternative by showing that two-dimensional distances in concept space (2Dxy) explain hippocampal responses significantly better than two-dimensional distance predictions that were derived by combinations with the irrelevant dimension [opacity-stripe frequency plane $(2 \mathrm{D} x z)$ or dot frequency-stripe frequency plane (2Dyz)].

Further, we ensured that the better fit of the two-dimensional conceptual distances cannot be attributed to weak memory of the conceptually irrelevant dimension. It should be noted that initial encoding of 3D associations took place before the conceptual relevance of two dimensions was introduced via the categorization task and should thus not be affected by this manipulation. To test memory as a result of all learning tasks at the time of scanning, participants were required to reconstruct the stimuli in all three feature dimensions after the postlearning fMRI session. Importantly, this $3 \mathrm{D}$ recall test revealed very high recall performance in all three dimensions (i.e., the average error below 1 step) and no difference in recall error across dimensions, ensuring that the third dimension was well encoded, even to degrees that are statistically equal to the conceptual dimensions. Although, differences between dimensions were not significant, the recall error for the conceptually irrelevant dimension was marginally higher. We consider this marginal difference unlikely to account for our pattern of results: the order of distance relations across object pairs in 3D (or alternatively 2Dxz/yz) feature space, would not be reversed by slight metric deviations on dimension $z$, leaving the respective feature-based distances a relatively appropriate prediction. Thus, if the only difference between the two conceptually relevant dimensions $(x, y)$ and the third dimension would be a slight difference in memory precision, 2D $x y$ distances should at most fit the hippocampal signal marginally better than distances in 3D (or alternatively, 2Dxz/yz); but one of these alternative models should have some explanatory power for the hippocampal signal on its own. Instead, we demonstrate that none of these alternative distance regressors that entails an integration of dimension $z$ can explain the hippocampal signal. Together, these results show that only the two dimensions that were relevant in relation (i.e., defining a concept) were integrated in a combined map-like representation, while the mnemonically, but not conceptually, relevant dimension was not. This suggests that the hippocampus can carve out (and represent) conceptual information from the totality of features, despite encoding specific exemplars in all detail.

As such, the present results help to elucidate the role of the hippocampus in concept learning. Previous studies suggested a role of the hippocampus in categorization (Nomura et al., 2007; Zeithamova et al., 2008; Davis et al., 2012; Mack et al., 2013;
Seger et al., 2015; Kim et al., 2018). How specific this involvement is with regard to the conceptual aspect of the task and how the hippocampus, as opposed to other brain regions, supports the acquisition of conceptual knowledge remained unclear. A recent proposal is that the spatial coding properties of the hippocampus and entorhinal cortex might be specifically suited to create representations that enable processes critical for the flexible use of knowledge such as inference and transfer (Behrens et al., 2018). The first experimental evidence demonstrated spatial coding principles in other cognitive domains (Tavares et al., 2015; Constantinescu et al., 2016; Aronov et al., 2017; Nau et al., 2018; Staudigl et al., 2018; Bao et al., 2019; Theves et al., 2019), and here we now link a spatial format of representation directly to concept learning. It has been proposed (Behrens et al., 2018) that while entorhinal grid cells might encode the structure of an environment (Constantinescu et al., 2016), the hippocampus encodes conjunctions of specific elements to this structure. This proposal would be congruent with the present finding (see also Theves et al., 2019) with the hippocampal representation reflecting the binding of specific objects in their conceptual context. It should be noted that the present results are not in contrast to the vast body of literature demonstrating cortically distributed representations of concepts embedded in long-term semantic knowledge (Martin, 2007, 2016; Binder and Desai, 2011; Ralph et al., 2017), but propose a spatial code for the formation of concepts in the hippocampus. Thus, while semantic information might ultimately be stored in neocortex, the hippocampus seems to critically support its acquisition (Kumaran, 2012; Elward and Vargha-Khadem, 2018). For instance, although patients with developmental amnesia because of hippocampal atrophy can show semantic memory comparable to that in control participants in everyday life (Vargha-Khadem et al., 1997; potentially compensated by direct cortical incorporation of new information into existing representations over time), the learning of completely new material (assumed to be critically supported by fast hippocampal processing of trial-unique stimuli) was shown to be ameliorated (Elward and Vargha-Khadem, 2018). Accordingly, a hippocampal organization of new information into a map-like format might support the acquisition of concepts, when fast extraction of critical relations or structures and commonalities across events is required. The present results suggest a role of the hippocampus in the formation of cognitive spaces spanned by relationally relevant feature dimensions, which provide sufficient flexibility for inferential processes or transfer, and from which more abstracted information (i.e., dichotomic category membership responses classically observed in PFC rather than hippocampus; Freedman et al., 2001, 2003; Wallis and Miller, 2003; Seger and Miller, 2010; Meyers et al., 2008; Roy et al., 2014) can be derived and coded by other brain regions. Accordingly, the present hippocampal representation reflects feature-based distances in a space spanned by conceptually relevant dimensions, without being driven by coarse category membership (post hoc analyses including category membership to the GLM reported above revealed that the $2 \mathrm{D}$ feature-based distance regressor remains significant $\left(t_{(31)}=1.983 ; p=0.0185\right)$, while category membership cannot explain the hippocampal signal $\left(t_{(31)}=-0.132\right.$, $p=0.552$ ). Instead, congruent with the conceptual nature of the present feature-based representation, post hoc analyses reveal that hippocampal responses to objects scales with the $2 \mathrm{D}$ distance of the objects to the category boundary $\left(t_{(31)}=1.704\right.$; $p=0.044$; inter-object distance was included as a regressor and remained significant: $\left.t_{(31)}=2.928, p=0.0015\right)$. As categories had been delineated via the diagonal through the two-dimensional feature space, information about the boundary emerges only from an integrated representation of conceptually relevant feature dimensions and can thus be considered further support for the spatial format of the representation.

In sum, by demonstrating that the hippocampus encodes distances between points in a concept space, as opposed to a full 
feature space, the present study provides critical evidence that hippocampal coding principles provide a suitable format to represent conceptual knowledge.

\section{References}

Aronov D, Nevers R, Tank DW (2017) Mapping of a non-spatial dimension by the hippocampal-entorhinal circuit. Nature 543:719-722.

Bao X, Gjorgieva E, Shanahan LK, Howard JD, Kahnt T, Gottfried JA (2019) Grid-like neural representations support olfactory navigation of a twodimensional odor space. Neuron 102:1066-1075.

Behrens TEJ, Muller TH, Whittington JCR, Mark S, Baram AB, Stachenfeld KL, Kurth-Nelson Z (2018) What is a cognitive map? Organizing knowledge for flexible behavior. Neuron 100:490-509.

Binder JR, Desai RH (2011) The neurobiology of semantic memory. Trends Cogn Sci 15:527-536.

Blair RC, Karniski W (1993) An alternative method for significance testing of waveform difference potentials. Psychophysiology 30:518-524.

Collin SH, Milivojevic B, Doeller CF (2015) Memory hierarchies map onto the hippocampal long axis in humans. Nat Neurosci 18:1562-1564.

Constantinescu AO, O’Reilly JX, Behrens TEJ (2016) Organizing conceptual knowledge in humans with a gridlike code. Science 352:1464-1468.

Davachi L (2006) Item, context and relational episodic encoding in humans. Curr Opin Neurobiol 16:693-700.

Davachi L, Mitchell JP, Wagner AD (2003) Multiple routes to memory: distinct medial temporal lobe processes build item and source memories. Proc Natl Acad Sci U S A 100:2157-2162.

Davis T, Love BC, Preston AR (2012) Striatal and hippocampal entropy and recognition signals in category learning: simultaneous processes revealed by model-based fMRI. J Exp Psychol Learn Mem Cogn 38:821-839.

Eichenbaum H (2004) Hippocampus: cognitive processes and neural representations that underlie declarative memory. Neuron 44:109-120.

Elward RL, Vargha-Khadem F (2018) Semantic memory in developmental amnesia. Neurosci Lett 680:23-30.

Freedman DJ, Riesenhuber M, Poggio T, Miller EK (2001) Categorical representation of visual stimuli in the primate prefrontal cortex. Science 291:312-316.

Freedman DJ, Riesenhuber M, Poggio T, Miller EK (2003) A comparison of primate prefrontal and inferior temporal cortices during visual categorization. J Neurosci 23:5235-5246.

Groppe DM (2010) One sample/paired samples permutation t-test with correction for multiple comparisons File Exchange, MATLAB Central. Natick, MA: MathWorks. Available at: http://www.mathworks.com/ matlabcentral/fileexchange/29782-one-sample-paired-samples-permutationt-test-with-correction-for-multiple-comparisons/content/mult_comp_ perm_t1.m.

Hafting T, Fyhn M, Molden S, Moser MB, Moser EI (2005) Microstructure of a spatial map in the entorhinal cortex. Nature 436:801-806.

Horner AJ, Bisby JA, Zotow E, Bush D, Burgess N (2016) Grid-like Processing of Imagined Navigation. Curr Biol 26:842-847.

Howard LR, Javadi AH, Yu Y, Mill RD, Morrison LC, Knight R, Loftus MM, Staskute L, Spiers HJ (2014) The hippocampus and entorhinal cortex encode the path and Euclidean distances to goals during navigation. Curr Biol 24:1331-1340.

Kemp C (2012) Exploring the conceptual universe. Psychol Rev 119:685-722.

Kim J, Castro L, Wasserman EA, Freeman JH (2018) Dorsal hippocampus is necessary for visual categorization in rats. Hippocampus 28:392-405.

Kim M, Jeffery KJ, Maguire EA (2017) Multivoxel pattern analysis reveals 3D place information in the human hippocampus. J Neurosci 37:4270-4279.

Knowlton BJ, Squire LR (1993) The learning of categories: parallel brain systems for item memory and category knowledge. Science 262:1747-1749.

Komorowski RW, Garcia CG, Wilson A, Hattori S, Howard MW, Eichenbaum H (2013) Ventral hippocampal neurons are shaped by experience to represent behaviorally relevant contexts. J Neurosci 33:80798087.

Kumaran D (2012) What representations and computations underpin the contribution of the hippocampus to generalization and inference? Front Hum Neurosci 6:157.

Mack ML, Preston AR, Love BC (2013) Decoding the brain's algorithm for categorization from its neural implementation. Curr Biol 23:2023-2027.
Mack ML, Love BC, Preston AR (2016) Dynamic updating of hippocampal object representations reflects new conceptual knowledge. Proc Natl Acad Sci U S A 113:13203-13208.

Martin A (2007) The representation of object concepts in the brain. Annu Rev Psychol 58:25-45.

Martin A (2016) GRAPES-Grounding representations in action, perception, and emotion systems: how object properties and categories are represented in the human brain. Psychon Bull Rev 23:979-990.

Meyers EM, Freedman DJ, Kreiman G, Miller EK, Poggio T (2008) Dynamic population coding of category information in inferior temporal and prefrontal cortex. J Neurophysiol 100:1407-1419.

Milivojevic B, Doeller CF (2013) Mnemonic networks in the hippocampal formation: from spatial maps to temporal and conceptual codes. J Exp Psychol Gen 142:1231-1241.

Milivojevic B, Vicente-Grabovetsky A, Doeller CF (2015) Insight reconfigures hippocampal-prefrontal memories. Curr Biol 25:821-830.

Morgan LK, Macevoy SP, Aguirre GK, Epstein RA (2011) Distances between real-world locations are represented in the human hippocampus. J Neurosci 31:1238-1245.

Nau M, Navarro Schröder T, Bellmund JLS, Doeller CF (2018) Hexadirectional coding of visual space in human entorhinal cortex. Nature Neuroscience 21:188-190.

Nomura EM, Maddox WT, Filoteo JV, Ing AD, Gitelman DR, Parrish TB, Mesulam M-M, Reber PJ (2007) Neural correlates of rule-based and information-integration visual category learning. Cereb Cortex 17:37-43.

O'Keefe J, Dostrovsky J (1971) The hippocampus as a spatial map. Preliminary evidence from unit activity in the freely-moving rat. Brain Res 34:171-175.

Porter BS, Schmidt R, Bilkey DK (2018) Hippocampal place cell encoding of sloping terrain. Hippocampus 28:767-782.

Ralph MA, Jefferies E, Patterson K, Rogers TT (2017) The neural and computational bases of semantic cognition. Nat Rev Neurosci 18:42-55.

Ranganath C (2010) Binding items and contexts: the cognitive neuroscience of episodic memory. Curr Dir Psychol Sci 19:131-137.

Roy JE, Buschman TJ, Miller EK (2014) PFC neurons reflect categorical decisions about ambiguous stimuli. J Cogn Neurosci 26:1283-1291.

Schlichting ML, Mumford JA, Preston AR (2015) Learning-related representational changes reveal dissociable integration and separation signatures in the hippocampus and prefrontal cortex. Nat Commun 6:8151.

Seger CA, Miller EK (2010) Category learning in the brain. Annu Rev Neurosci 33:203-219.

Seger CA, Braunlich K, Wehe HS, Liu Z (2015) Generalization in category learning: the roles of representational and decisional uncertainty. J Neurosci 35:8802-8812.

Smith EE, Medin DL (1981) Categories and concepts. Cambridge, MA: Harvard UP.

Staudigl T, Leszczynski M, Jacobs J, Sheth SA, Schroeder CE, Jensen O, Doeller CF (2018) Hexadirectional modulation of high-frequency electrophysiological activity in the human anterior medial temporal lobe maps visual space. Curr Biol 28:3325-3329.

Tavares RM, Mendelsohn A, Grossman Y, Williams CH, Shapiro M, Trope Y, Schiller D (2015) A map for social navigation in the human brain. Neuron 87:231-243.

Theves S, Fernandez G, Doeller CF (2019) The hippocampus encodes distances in multidimensional feature space. Curr Biol 29:1226-1231.

Vargha-Khadem F, Gadian DG, Watkins KE, Connelly A, Van Paesschen W, Mishkin M (1997) Differential effects of early hippocampal pathology on episodic and semantic memory. Science 277:376-380.

Wallis JD, Miller EK (2003) From rule to response: neuronal processes in the premotor and prefrontal cortex. J Neurophysiol 90:1790-1806.

Wohlgemuth MJ, Yu C, Moss CF (2018) 3D hippocampal place field dynamics in free-flying echolocating bats. Front Cell Neurosci 23:270.

Yartsev MM, Ulanovsky N (2013) Representation of three-dimensional space in the hippocampus of flying bats. Science 340:367-372.

Zaki SR (2004) Is categorization performance really intact in amnesia? A meta-analysis. Psychon Bull Rev 11:1048-1054.

Zeithamova D, Maddox WT, Schnyer DM (2008) Dissociable prototype learning systems: evidence from brain imaging and behavior. J Neurosci 28:13194-13201. 